\title{
Simulation of an Accelerator Driven Subcritical Core with Mixed Uranium-Thorium Fuel
}

\author{
Ali Pazirandeh, Laia Shirmohammadi \\ Department of Nuclear Engineering, Science and Research Branch, Islamic Azad University, Tehran, Iran \\ Email: pzrud193y@srbiau.ac.ir
}

Received 17 May 2015; accepted 23 October 2015; published 30 October 2015

\begin{abstract}
During recent years, a new generation of nuclear reactors, known as "Accelerator Driven Subcritical Reactors", has been developed. One of the new application aspects for such reactors (besides transmutation of High Level Waste and burning Minor Actinides) is usage of thorium as nuclear fuel. In this work a subcritical core in experimental scale is simulated by MCNPX code. The core contains two types of fuel assemblies: $\left(85 \% \mathrm{ThO}_{2}+15 \% \mathrm{UO}_{2}\right)$ and MOX (U-Pu). In the first step, only the thorium-contained fuel assemblies are loaded into the core. Criticality calculations using MCNPX show that the $k_{\text {eff }}$ is so low that the fuel assemblies cannot run the subcritical core. This implies that MOX (U-Pu) assemblies must be loaded as well. Neutronic parameters of the thoriumfueled Accelerator Driven Subcritical core are then calculated as well as some other parameters related to accelerator coupled with the core. The main objective of this simulation is to study the behavior of Accelerator Driven Subcritical core with thorium assemblies.
\end{abstract}

\section{Keywords}

Accelerator Driven Subcritical Reactor, Thorium, MCNPX, Neutronic Calculation

\section{Introduction}

Accelerator Driven Systems (ADS) attract worldwide attention owing to their superior aspects and their ability to burn Minor Actinides (MA) and fission products in conjunction to their ability to transmute HLW and to produce energy. Furthermore, ADS benefit from vitally important advantage of employing thorium (as a new generation of nuclear fuel) with drastically wider safety margin compared with that of uranium. Thorium is an actinide, metallic element whose abundance in earth's crust is $6 \mathrm{ppm}$ (i.e., three times more than that of uranium) and is found naturally on the earth only as ${ }^{232} \mathrm{Th}\left(\mathrm{t}_{1 / 2}=1.4 \times 10^{10} \mathrm{y}\right)$ [1]. Thorium has several advantages as a nuclear fuel:

It produces less of the nuclear by-products normally used to make nuclear weapons and less of the long-lived radioactive products of conventional nuclear power Its uses in suitable nuclear reactors can reduce the hazard of nuclear accidents unlike natural uranium, Its energy content can be used almost in its entirety, and thorium ore 
minerals are abundantly available in many countries including, Australia, India, ...

Accelerator Driven Thorium Reactor (ADTR) has some special properties that make it different from classic reactors. All AD systems consist of three main components: a Subcritical Core (SC), a spallation targets (ST) and an accelerator coupled with SC. Physics of AD reactors, in both structure and operation method, is quite different from other reactors. No control rod is used in these systems; instead, a proton beam from the accelerator coupled with SC controls and handles the core. This implies that the safety of the ADS basically depends on the function of the accelerator [2]-[4].

\section{Accelerator Driven Subcritical Core Simulation by MCNPX Code [5]}

The subcritical system adopts a hexagonal fuel array in order to compact the core and to achieve hard neutron energy spectrum by minimizing neutron moderation. The core is simulated using Monte Carlo code MCNPX 2.4.0. The core includes a cylindrical accelerator driven subcritical system loaded with $\left(85 \% \mathrm{ThO}_{2}+15 \% \mathrm{UO}_{2}\right)$ and MOX (U-Pu) fuel assemblies. The MOX (U-Pu) nuclides can be discharged from highly burnt-up spent fuel assemblies of a PWR. The core consists of 66 hexagonal $\left(85 \% \mathrm{ThO}_{2}+15 \% \mathrm{UO}_{2}\right)$ fuel assemblies, $54 \mathrm{MOX}$ (U-Pu) fuel assemblies, 90 reflector assemblies and 54 shield assemblies (see Figure 1). Each assembly comprises 37 rods. The radius of accelerator tube is $2.5 \mathrm{~cm}$ and $32.6 \mathrm{~cm}$ below the top of subcritical core. There is no control rod in the core. The subcritical core is generally driven by external neutrons emerged from the spallation target that is bombarded by charged particles accelerated and collimated in a high-power accelerator. Here, the system is driven by $1 \mathrm{GeV}$ proton beam aimed to LBE (Lead-Bismuth Eutectic) target. The structure walls and beam window are made of stainless-steel (HT-9). Owing to its outstanding nuclear properties, liquid leadbismuth $(44.5 \% \mathrm{~Pb}+55.5 \% \mathrm{Bi})$ is employed as both coolant and spallation target. Low melting point, high boiling point and very low vapor pressure of LBE make it proper to operate within a wide range of temperature that implies an extraordinary natural convection and cooling melting point, high boiling point and very low vapor pressure of LBE make it proper to operate within a wide range of temperature that implies an extraordinary natural convection and cooling capability leading to an enhanced passive safety. Because of its low neutron capture cross section, LBE is transparent to neutrons, allowing much relaxed core design. The configuration of the core is illustrated in Figure 1.

The shield is made of $\mathrm{B}_{4} \mathrm{C}$. Figure 2 shows a cross-sectional view of a single assembly. The structural assembly walls are made of stainless-steel (HT-9). No separate target is included in the core. As depicted in Figure 1, the buffer region is between LBE target and the core. The buffer zone flattens the power distribution. Figure 3 demonstrates the array of assemblies in the core simulated by MCNPX2.4.0.

\section{The Neutronic Performance Calculation and Results for Simulated ADS Core}

The calculation of neutron multiplication factor for ADS was performed by MCNPX code using KCODE option. In an ADS, $k_{\text {eff }}$ must be between 0.95 and 0.98 indicating that ADS is a subcritical system. ADS reactors, as a matter of fact, are subcritical in all conditions and power levels. The system was first simulated with only (Th-U) fuel assemblies loaded and the multiplication factor was calculated. This being the case, $\mathrm{k}_{\text {eff }}$ was much less than 0.95, implying that the core would not run. Then the MOX (U-Pu) fuel assemblies were loaded into the core as shown in Figure 1 and the multiplication factor increased to 0.966 where the chain reaction could sustain. The

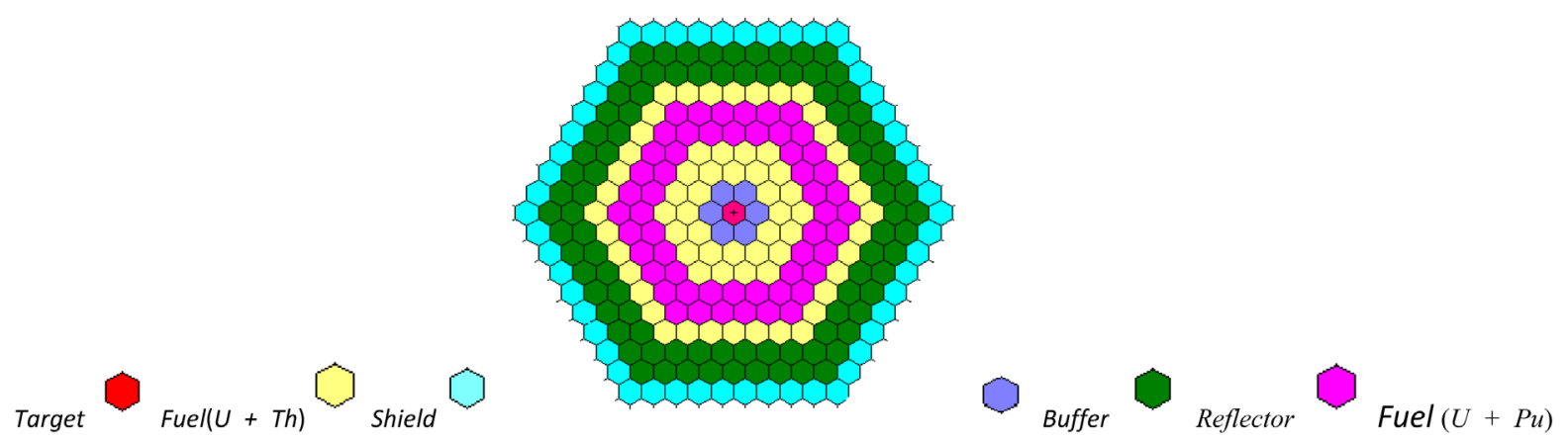

Figure 1. Cutaway of simulated ADS core. 


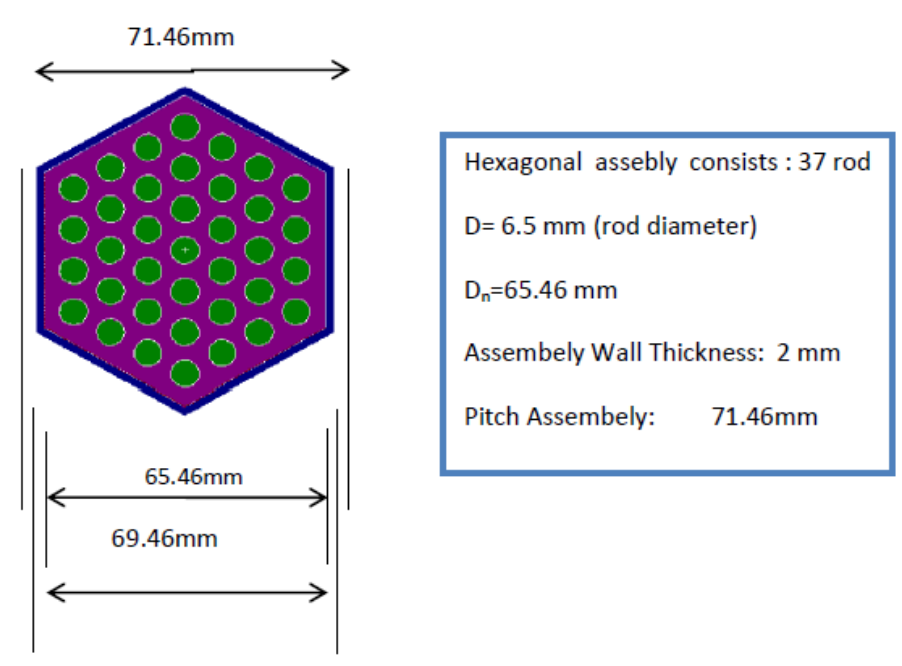

Figure 2. Cutaway of an assembly.
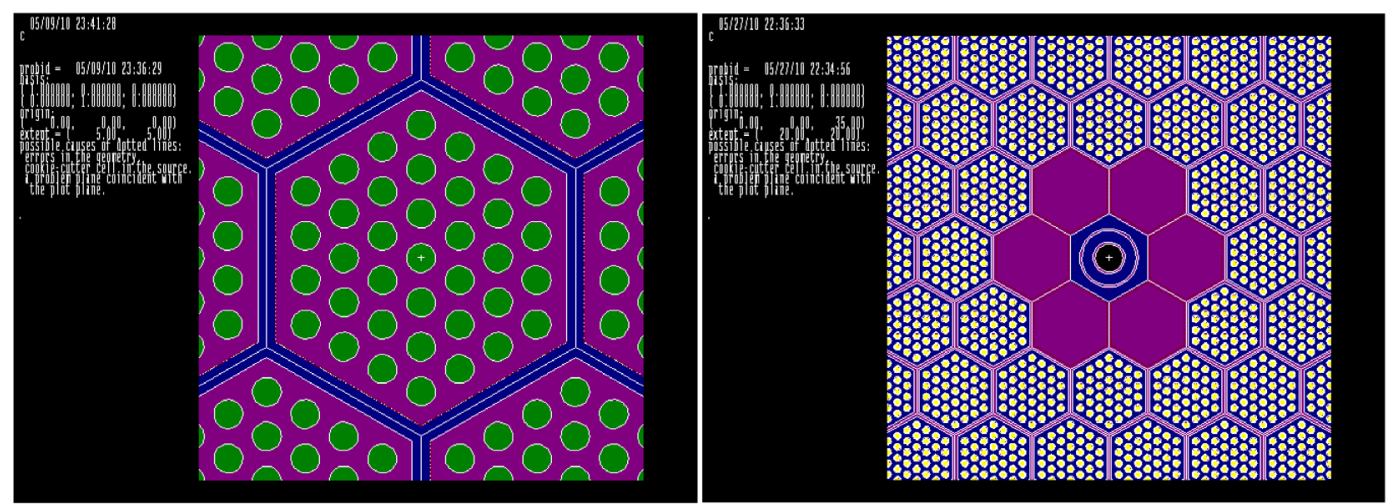

Figure 3. Cutaway of assembly array in the core simulated by MCNPX: (a) one assembly (b) part of the core assemblies, buffer region and target.

spallation neutrons were produced from interaction between $1 \mathrm{GeV}$ proton beam and LBE target. A critical system (in which neutron production and consumption are balanced) is well defined in the phase space $(\mathrm{E}, \boldsymbol{r}, \boldsymbol{\Omega})$ by Boltzmann equation:

$$
A \Phi=\mathrm{P} \Phi
$$

In the subcritical system the condition to have a stationary state is to have an external source $\mathrm{S}(\mathrm{E}, \bar{r}, \bar{\Omega})$ so Equation (1) changes to:

$$
A \Phi_{\text {in }}=P \Phi_{\text {in }}+S
$$

$\Phi_{i n}$ is the solution of the inhomogeneous Equation (2). Relevant integral parameters characterizing the subcritical core is done exactly as in critical systems, characterized by, is formally possible to describe a subcritical system with the introduction of a parameter $k_{e f f}$ as given in Equation (3).

$$
A \Phi=\frac{1}{k_{\text {eff }}} P \Phi
$$

To improve the definition of sub-criticality a different definition of the sub-criticality has been proposed, by means of a k-source " $k_{S}$ " the external source multiplication factor one obtains:

$$
k_{S}=\frac{\left\langle P \Phi_{\text {in }}\right\rangle}{\left\langle P \Phi_{\text {in }}\right\rangle+\langle S\rangle}
$$


$k_{S}$ depends on the incident proton energy, target material and dimension of the target. Therefore, in ADS safety and dynamic development accelerator technology with high energy beams in parallel with subcritical core study appears necessary. In ADS core, $k_{S}=(M-1) / M$ and $M$ is the number of neutrons produced per proton, so for simulated core, in the top $\mathrm{Z}=32 \mathrm{~cm}$ of core $\mathrm{M}=27.53$ is approached. The relative importance of the source neutrons to the fission neutrons generated in the SC is introduced by a parameter $\Phi^{*}$ as neutron source importance is related to $k_{\text {eff }}$ as:

$$
\frac{\Gamma}{v} \varphi^{*}=\frac{1}{k_{\text {eff }}}-1
$$

where $\bar{v}$ is the average of prompt neutrons per fission and $\Gamma$ is the average number of source neutrons per fission.

Another equation for this parameter is:

$$
\varphi^{*}=\left(\frac{1}{k_{\text {eff }}}-1\right) /\left(\frac{1}{k_{S}}-1\right)
$$

The $\varphi^{*}$ parameter plays an important role in assessing the ADS performance parameters. For this simulated core $\varphi^{*}$ is 0.967 . Table 1 shows some data related to simulated core.

Figure 4 shows the variation of neutron yield per incident proton versus the energy of the proton in target region. The neutron yield is calculated by simulating an LBE cylindrical target outside the core and an incident proton beam. Neutrons in the both types of fuel assemblies are fast, implying that the simulated core behaves like a fast reactor core. The neutron spectra in shielding, $\operatorname{MOX}(\mathrm{U}+\mathrm{Th})$, buffer and $(\mathrm{U}+\mathrm{Pu})$ regions are shown in Figure 5.

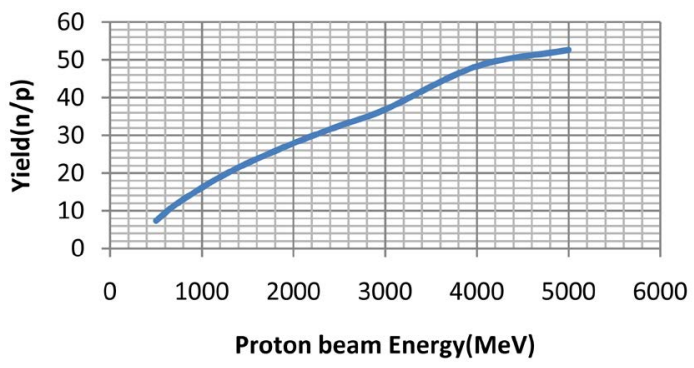

Figure 4. Variation of neutron yield per incident proton beam in target.

Table 1. Data of the simulated accelerator driven subcritical core.

\begin{tabular}{cc}
\hline$k_{\text {eff }}$ (multiplication factor) & 0.966 \\
$k_{\mathrm{s}}$ (external source multiplication factor) & 0.965 \\
$\mathrm{M}$ (the number of neutron produce per proton beam) & 27.53 \\
$\rho$ (reactivity) & -0.0351 \\
$\varphi^{*}$ (adjoint flux) & 0.9673 \\
G (energy gain) & 60.8 \\
Core thermal power & $44.49 \mathrm{MW}$ \\
Peaking factor (total core) & 1.56 \\
Cycle length & $300 \mathrm{~d}$ \\
Av neutron energy & $190 \mathrm{KeV}$ \\
Av neutron flux & $3.56 \mathrm{e} 15\left(\mathrm{n} / \mathrm{cm}^{3} \mathrm{~s}\right)$ \\
Active core hight & $100 \mathrm{~cm}$ \\
\hline
\end{tabular}




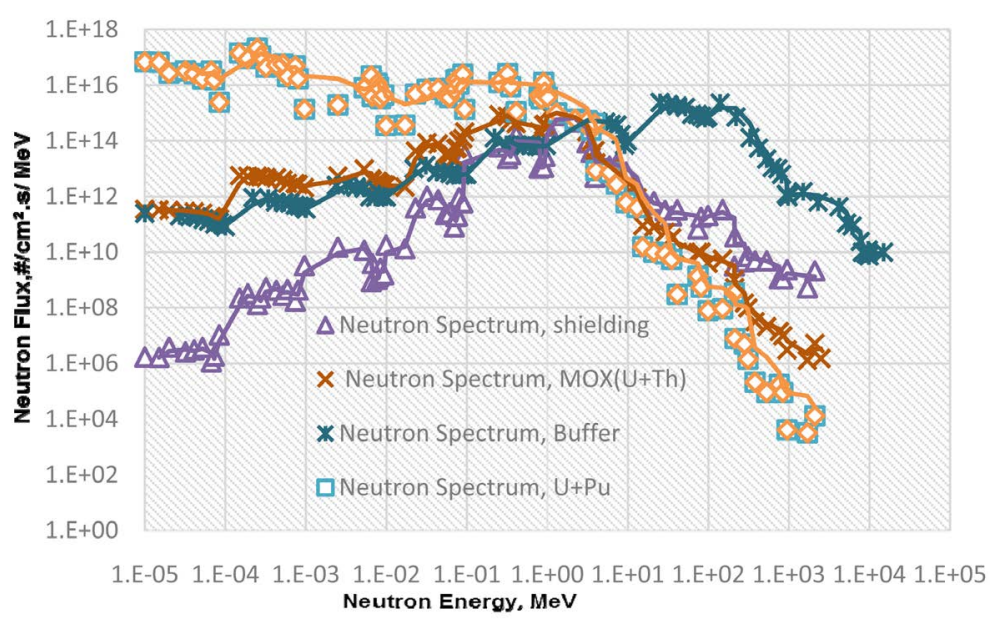

Figure 5. Neutron flux in buffer region.

\section{Conclusions}

Why we should use thorium instead of uranium?

Because of simply reason, it involves nuclear reactions that are much more effective than those in conventional nuclear reactors. Thorium is about three times more abundant on Earth than uranium; it is about as abundant as lead. Another advantage of thorium is that it is less of a proliferation threat-there is a uranium bomb and a plutonium bomb, but no thorium bomb can be produced. The use of thorium has received a lot of attention from many scientists, such as Alvin Weinberg, Ed Teller and Carlo Rubbia. But the main drawback with thorium is that you need two neutrons, rather than the one in ordinary uranium powered reactions, to produce we need to use a proton accelerator It is a problem that demands $1-1.5 \mathrm{GeV}$ accelerator. To produce fissile isotope is shown in reaction below:

$$
\mathrm{N}+{ }^{232} \mathrm{Th} \rightarrow{ }^{233} \mathrm{Th} * \rightarrow{ }^{233} \mathrm{~Pa}+\beta^{-} \rightarrow{ }^{233} \mathrm{U}+\beta^{-}
$$

Researches on thorium fuel cycle are currently underway and thorium-fuelled reactors must be brought into the service to let their technological aspects be practically investigated. ADS reactor is a new generation, hightech system that can burn thorium-based fuel to produce power. This work simulates an ADS core having two different types of fuel assemblies and burning thorium fuel. ADS systems produce less nuclear wastes, eliminating the complicated problem confronted in the nuclear waste management. The new generation of nuclear fuel (e.g., Minor Actinides (MA) and MOX (Th-U) or MOX (Th-Pu)) needs no uranium enrichment and no plotuinum, therefore, is much more economic. The accelerator that drives the system plays a very essential role in technological and safety aspects. Further studies on the proton beams produced by the accelerator and on the interactions of proton with matter may help to choose better materials as the spallation target and to optimize the neutron flux emerged from the external source. In Accelerator Driven Thorium Reactors (ADTR), on the other hand, very rare radio-isotopes may be produced for medical application [6]. As pointed out the multiplication factor ought to be greater than 0.95, since for an ADS with a neutron multiplication factor of 0.95, the reduction amounts to about $12 \%$. This means that the accelerator driven system produces about $14 \%$ more high-level waste and rejects about $20 \%$ more heat to the atmosphere than a normal power plant with the same net electrical output. The advantages and disadvantages of accelerator-driven systems as compared with corresponding critical reactor systems applies not only to transmutation applications, but also to other applications such as the breeding of fissile material, transmutation of long lived fission products, the development of the 233Th to 233U fuel cycle, and the development of ultra-safe energy producers [7]. Extended neutron energy spectrum promotes fast fission reactions in actinides. Transmutation aims at reducing the radiological impact of actinides and fission products in the HLW by nuclear transformation of troublesome long-lived nuclides in strong radiation fields. The application of the ADS concept to a minor actinide burner core is an interesting possibility to compensate the safety disadvantages arising from the small Doppler coefficient and the small $k_{\text {eff }}$ value which cannot be otherwise compensated. 


\section{References}

[1] IAEA Headquarters (2010) Review Benchmarking of Nuclear Data for the Th-U Fuel Cycle. IAEA Headquarters Vienna, Austria, INDC (NDS), 586.

[2] Degweker, S.B., Satyamurthy, P., Nema, P.K. and Singh, P. (2007) Program for Development of Accelerator Driven System in India. Pramma Journal of Physics, 68, 257-268.

[3] Hashemi-Nezhad, S.R. (2006) Accelerator Driven Subcritical Nuclear Reactor for Safe Energy Production and Nuclear Waste Incineration. Australian Physics, 43, 90-96.

[4] Rubens, M.J. and Thiago, C. (2004) A Review of Thorium Utilization as an Option for Advanced Fuel Cycle Potential Option for Brazilian Future. ANES, American Nuclear Energy Symposium, Miami Beach, 3-6.

[5] Briesmeister, J.F. (2000) MCNPX2.4.0: MCNP_A General Monte Carlo N-Particle Transports.

[6] Juraj, B., Petr, D. and Vladimir, N. (2010) Study of Thorium Advanced Fuel Cycle Utilization in Light Water Reactor VVER-440. Annals of Nuclear Energy, 37, 685-690. http://dx.doi.org/10.1016/j.anucene.2010.02.003

[7] Sylvian, D., Elisabeth, H. and Herve, N. (2007) Revisiting the Thorium-Uranium Nuclear Fuel Cycle. Euro-Physics News, 38, 2. 